\title{
Spinal Cord Analysis in People with Multiple Sclerosis
}

\author{
${ }^{1}$ Fatemeh Ebrahimi Meymand, ${ }^{2}$ Hasan Daneshmandi ${ }^{*},{ }^{3}$ Mansoor Sahib Al-Zamani, \\ ${ }^{4}$ Hossein Ali Ebrahimi
}

\begin{abstract}
${ }^{1}$ Department of Sport Injury and Corrective Exercise, Faculty of Sport Sciences, Kish International Campus, Tehran University, Kish, Iran. ${ }^{2}$ Department of Sport Injury and Corrective Exercise, Faculty of Sport Sciences, University of Guilan, Rasht, Iran. ${ }^{3}$ Department of Sport Injury and Corrective Exercise, Faculty of Sport Sciences, Shahid Bahonar University, Kerman, Iran. ${ }^{4}$ Neurology Research Center, Kerman University of Medical Sciences, Kerman, Iran.
\end{abstract}

\begin{abstract}
Background. Multiple Sclerosis (MS) is an autoimmune, inflammatory, and chronic disease which, under the effect of the wastes of myelin degradation occurs in the white matter of the brain, spinal cord, and visual nerves. The main complications of this disease are fatigue, muscle cramps, tremor, imbalance, and walking imbalance. Objectives. The purpose of this study is to investigate the spine orientation in people with multiple sclerosis. Methods. The statistical population of this study included all patients with MS in Kerman province and the sample of this study includes 30 patients with mild MS (mean age of $33.83 \pm 8.72$ years, mean height of $159.77 \pm 4.54 \mathrm{~cm}$, mean weight of $63.1 \pm 11.16$ $\mathrm{kg}$ ) and 30 healthy subjects (mean age of $31.07 \pm 8.84$ years, mean height of $162.58 \pm 6.61 \mathrm{~cm}$, mean weight of $61.43 \pm 11.3 \mathrm{~kg}$ ), targeted randomly selected as the subjects. First, the patients were examined by a neurologist and the EDSS of each subject was evaluated. Then their demographic data including age, height, and weight were collected. For evaluation of spinal abnormalities including torticollis and forward head, shooting method with the use of -Power shot A1200 HD Canon camera, with 0.73 validity was used; and kyphosis and lordosis abnormalities were measured using a flexible ruler, the validity of the device in comparison with the radiography technique was reported by Thylatson and Borton (1991) as 93\%. In research, Hart \& Rose (1986) and Lowell et al. (1989) stated the reliability of this device as $97 \%$ and $84 \%$, respectively, and scoliometer was used for scoliosis abnormalities. The validity of this device was reported by Guillemhem et al. as 0.87 . Results. The results showed that there was a significant relationship between forward head, torticollis, and lordosis abnormalities in patients with mild MS and healthy subjects $(\mathrm{p}<0.05)$, while there is significant relationship between kyphosis and scoliosis $(\mathrm{p}<0.05)$. Conclusion. Regarding factors such as fatigue, muscle cramping, tremor, walking abnormalities in people with multiple sclerosis and observation of some spinal abnormalities in these individuals, the implementation of corrective exercises and programs to correct postures, in them, seems necessary.
\end{abstract}

KEY WORDS: Spine, Postural Profile, Multiple Sclerosis (MS).

\section{INTRODUCTION}

Chronic diseases are addressed as the main challenge of health systems in the twenty-first century. Today, in terms of epidemiology, noncommunicable diseases are responsible for $60 \%$ of the world's death and mortality cases (1). Among these diseases, diabetes, asthma, multiple sclerosis etc. could be mentioned (2). Multiple sclerosis is a chronic inflammatory disease

*. Corresponding Author:

Hasan Daneshmandi

E-mail: daneshmandi_ph@yahoo.com 
characterized by the demyelin wastes in the brain, spinal cord, and visual nerves (3). This disease is a major cause of neurodevelopment in young and middle-aged people. Today, about 2.5 million people in the world are suffering from MS; according to the statistics of the Iranian Multiple Sclerosis Society, there are 60,000 patients suffering from MS in the country $(4,5)$. Today, several methods are used to evaluate MS patients, the largest measure used to assess MS patients' disabilities is EDSS. At present, EDSS, Kurtzke has been accepted as a standard measure to assess the progression of MS, and has replaced other methods since it evaluates the patient's disability as compared with other methods and criteria in a wider context (6).

In this disease, myelin sheaths are damaged in the neural cells in the brain and spinal cord. This impairment affects the ability of parts of the nervous system that are responsible for communication, causing symptoms and physical problems $(7,8)$.

The complications of this disease include: 1) fatigue, which is one of the most important reasons why patients do not participate in physical activity; 2. Defect in controlling the condition of the body, they cannot directly hold their postures (9); 3. Walking disturbances, since MS patients gradually decrease their walking speed, and their step length decreases as well, these two factors lead to balance decrease and eventually non-control of posture (10); 4 . The weakness of the Hamstring muscles and the quadriceps in these people cause disturbances in walking and thus reduce postural stability (11, 12).

Some studies conducted on patients with MS, showed that the slightest change in the physical condition of these patients leads to postural instability, and changes in their physical condition has more fluctuation and requires more time to achieve stability than the control group $(13,14)$, and postural control when standing in people with mild to moderate MS is defective (15). Besides, forward head leads to headaches, neck pain, and pain between two scapula (16). Patients with MS always complain of pain between the two scapula and neck pain.

Also, the effects of fatigue and pain can reduce the natural movement of humans and reduce the effectiveness of various apparatus, especially the musculoskeletal system. Due to this decrease in mobility and activity, the motor range of joints in various parts of the body is reduced, and as time goes on, the individual experiences a reduction in flexibility, muscle weakness, loss of strength and muscular endurance, which together causes a variety of pain and discomfort in various parts of the body, especially the neck (17-19). Patients with MS have disturbances in the neck region and are exposed to kyphoscoliosis due to the muscular imbalance, resulting in a severe pressure on nerves (19). Also, neck pain in patients with MS is more than the pain in the trunk and back (20).

Studies have shown that patients with MS encounter myopathy disturbance in the neck in the spinal column. It is possible that patients with MS encounter kyphoscoliosis due to lack of muscular balance, which can cause unusual stress to the neural roots. Neck pains can also have an effect in the inconsistency of the upper limbs and the trunk $(19,21)$.

Since MS patients suffer from fatigue and muscle weakness, it causes changes in the muscle chain, and these negative changes can apply negative changes on the nervous and skeleton system too, and ultimately tends to make the body susceptible to changes in the physical condition. Muscular imbalance in these individuals increases the probability of anomalies in the spine (1). For example, because they have walking disturbance, and they often look at their steps to eliminate it, hence, there is the probability of torticollis and forward head in the neck, the neck kyphosis in the back spine, and lordosis in the spinal cord, also there is the probability of scoliosis in the spinal cord. Therefore, this study attempts to examine the postural profile of the spine cord and the anthropometric dimensions of MS patients to find out the degree of abnormalities in the condition that results from the disease.

\section{MATERIALS AND METHODS}

Participants. The subjects of this study included 30 women with mild MS with EDSS scale of 3 and lower, who visited the centers of specific diseases of Kerman. The incidence rate of MS in Kerman was 31.5 persons per 100,000 (6).

Instruments and Procedure. For measuring forward head, imaging with the use of the Power 
shot camera A1200 HD canon camera was used. First, the $\mathrm{C} 7$ cervical spine and Tragus are marked with the marker, then image is taken from one side of the subject, and in the AutoCAD software, the degree of the angle between them with the vertical line passing through the $\mathrm{C} 7$ bead is obtained. To measure the torticollis, the angle between the right and left ear lines with the horizon is determined by imaging and its angle is determined through the AutoCAD software; the normal angle of this abnormality is 46 degree (figure 1) (22).
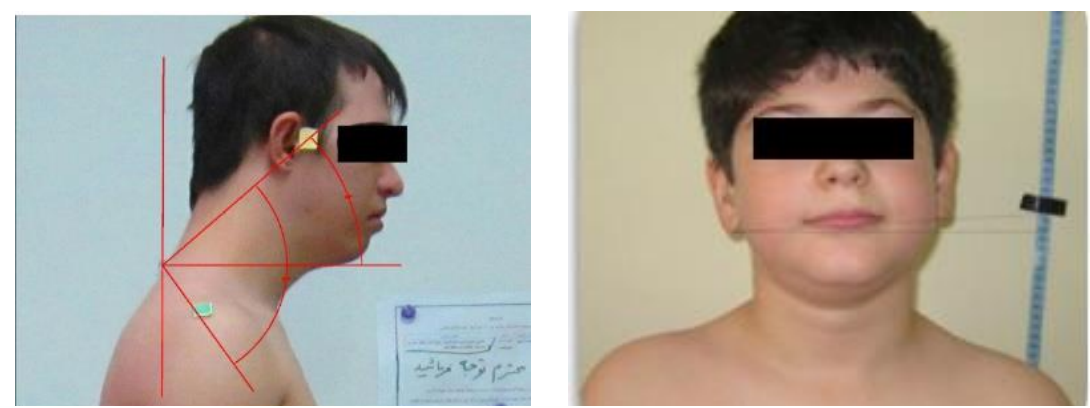

Figure 1. Measurement of forward head.

To measure kyphosis and lordosis, a flexible ruler was used whose reliability was confirmed by Hart \& Rose (1986) and Lowell et al. (1989) as 0.97 and 0.84 , respectively. Measures of the lumbar and back arches were done as follows: first, the subjects of the shock appendages of $\mathrm{T}_{2}, \mathrm{~T}_{12}, \mathrm{~L}_{1}$, and $\mathrm{S}_{2}$ were determined by the touch of the hands of the examiner. The subjects were asked to stand up and look forward in a comfortable state (23). Then the flexible ruler was placed between the second and the twelfth thoracic spine and then on the second lumbar spine and the second sacral spine on the shock appendages. The points of the second and the twelfth thoracic spine and the second lumbar spine and the second sacral spine were connected to one another with a straight line. This line is shown with $L$ in the formula. Then the perpendicular bisector to the arc was plotted shown with $h$ in the formula, and thus, the shape of the arc of kyphosis and lordosis was obtained and the mean lordosis angle is 50.9 degrees $\Theta=4$ $\arctan (2 \mathrm{~h} / \mathrm{L})$. (figure 2) (23).

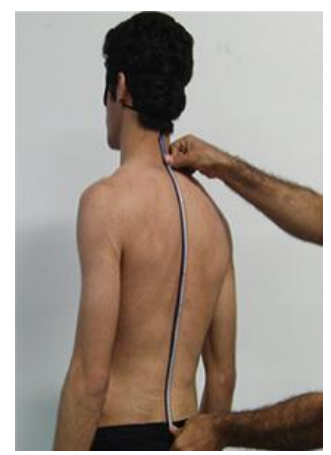

Figure 2. Measurement of kyphosis and lordosis.

To evaluate scoliosis, a digital scoliometer was used; the subject without a topcoat up bent forward until the upper body was level with shoulders and pelvis, and then he was imaged from the posterior perspective. The scoliometer was placed perpendicular to the body along the abnormality, so that its zero sign is placed on the tip of the sacral appendage. The scoliometer should be relied on the skin and should not be pressed down and then the number of degrees of rotation is recorded. If the angle obtained by the 
scoliometer at each level of the spine is 7 degrees or more, the result of the screening or examination is considered as positive (figure 3 ) (24).

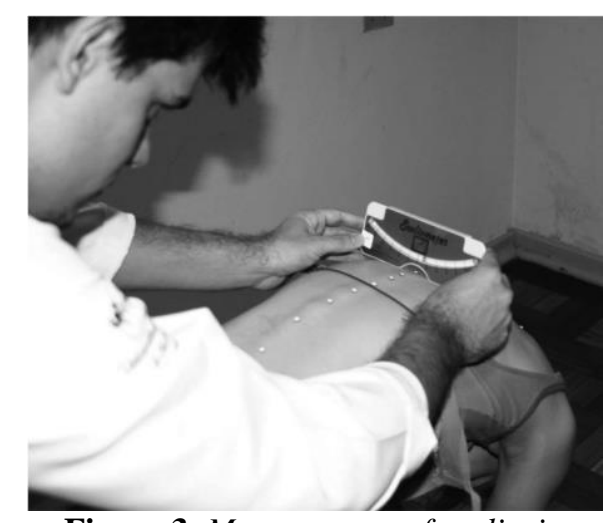

Figure 3. Measurement of scoliosis.

Statistical Analysis. For data analysis, SPSS software version 22 and independent $t$ test were used at the significance level of $\mathrm{p}<0.05$.

\section{RESULTS}

In this study, conducted on 30 women with mild MS with EDSS of 3 and lower, and 30 healthy women, where the age of women with mild MS was at least 21 up to 49 years of age; and the least age of healthy women was 20 up to 48 years of age (Table 1).

In order to determine the difference between forward head, torticollis, scoliosis, lordosis, and kyphosis abnormalities among the women with mild MS and healthy women, independent t-test was used. As shown in Table 2, there was a significant difference between the angle of forward head women with mild MS and healthy women $(\mathrm{p}<0.005)$. Also, the results of this study showed that the angle of torticollis in women with MS was significantly different from that of healthy women and the results showed a significant difference in angles in women with MS and healthy women $(\mathrm{p}<0.001)$. However, there was no statistical difference between scoliosis and kyphosis in women with MS and healthy women $(\mathrm{p}<0.07)$.

Table 1. Demographic data of the women with MS and the group of healthy women

\begin{tabular}{ccc}
\hline Variable & Women with MS & Healthy women \\
\hline Age $($ year $)$ & $33.83 \pm 8.72$ & $31.07 \pm 8.84$ \\
Height $(\mathrm{cm})$ & $159.77 \pm 4.54$ & $162.58 \pm 6.61$ \\
Weight $(\mathrm{kg})$ & $63.1 \pm 11.16$ & $61.43 \pm 11.03$ \\
BMI $\left(\mathrm{kg} / \mathrm{m}^{2}\right)$ & $33.27 \pm 4.11$ & $24.37 \pm 4.16$ \\
\hline
\end{tabular}

Table 2. comparison of forward head, torticollis, scoliosis, lordosis, and kyphosis in healthy and MS women

\begin{tabular}{cccccc}
\hline Variable & Women with mild MS & Healthy women & t & Df & p \\
\hline Forward head & $48.02 \pm 4.92$ & $44.6 \pm 4.57$ & -2.94 & 58 & $0.005 *$ \\
\hline Torticollis & $176.17 \pm 1.97$ & $178.83 \pm 1.58$ & -5.79 & 58 & $0.001 *$ \\
\hline Scoliosis & $1.54 \pm 1.6$ & $0.97 \pm 1.13$ & 1.81 & 58 & 0.70 \\
\hline Lordosis & $57.68 \pm 9.63$ & $49.3 \pm 9.63$ & 3.56 & 58 & $0.001 *$ \\
\hline Kyphosis & $29.1 \pm 9.14$ & $27.31 \pm 9.97$ & 0.73 & 58 & 0.47 \\
\hline & $*:$ significant at $\mathrm{p}<0.05$. & &
\end{tabular}




\section{DISCUSSION}

The results of this study showed that people with multiple sclerosis in comparison with healthy people, have some abnormalities, such as torticollis, forward head, and lordosis. In this disease, the distribution of force and pressure on the surface of the tissues has changed and leads to anomalies in the postural profile (3). Muscle fatigue and weakness are common in these patients. $80 \%$ of patients with MS suffer from fatigue and its effects on routine affairs, and some of these patients lose their jobs because fatigue reduces the ability of individuals to perform their individual and social tasks and roles, work and activity, and maintaining a natural life and lead to posture weakness in the individual (25). In patients with MS, probably due to some factors such as muscle weakness, reduced balance, visual acuity symptoms, hearing difficulties and neurological injuries, the risk of abnormalities is increased (25). Neurological disorders and neurological injuries are associated with reduced balance and ultimately postural weakness; inflammation of the visual nerve usually occurs in the patient MS in the form of blurred vision, pain and burning of the eyeball, and reduced of sharpness. Due to visual acuity in people with MS, these people tend to move their heads to a better position, so as to observe the object, in a smaller distance. This reduces neck which reduces the neck angle and temporal articulation disorders, shortness of the anterior cervical muscles and weakening of the cervical muscles of the mammary gland and ladder (6). Hamstring muscle weakness in MS patients results in abnormality in walking and thus reducing postural stability (12). The hamstring and gluteal muscle weakness can lead to lower interstitial and imbalance syndrome; this imbalance can have harmful effects on the static and dynamic status of the body, especially when walking. This syndrome increases the anterior pelvic tilt and in the spine increases lumbar lordosis and a small hip flexion $(1,11)$

It is reported that that MS patients suffer from postural and physical impairment, they cannot directly maintain their postures, which, as a result of this defect, disabilities are increased in posture and spinal column (9).
There are little research on spinal cord abnormalities in MS patients and healthy partners. Where except for the study by Mullen, Wilmarth, and Lowe (2012) which has been done regarding the postures of people with MS (19), other researches have been carried out in other people in the community, and this study is consistent with the studies conducted by Huisinga et al., 2012, Kasser and Jacobs, $2014(18,21)$.

Symptoms of MS include fatigue, weakness, visual acuity, neurological damage and ataxia (25). It is believed that sensory systems such as vision have an important role in controlling human movement, therefore, visual impairment can lead to problems with balance, posture, and coordination (4).

Mullen, Wilmarth, and Lowe (2012) said MS patients have disturbances in the neck area, hence the ability of these people to head control decreases and leads to abnormalities in the neck (19). So that, the results of the present study in MS patients compared to healthy subjects, both forward head and torticollis abnormality were significant. Also, Mullen, Wilmarth, and Lowe (2012) stated in their research that because of muscle imbalance, these individuals are infected with kyphoscoliosis, which results in a lot of pressure on nerves and the results of the present study did not match this abnormality (19); the results of this study showed that this people did not suffer from abnormalities of kyphosis and scoliosis. Another study reported that due to changes in the physical condition as a result of lower interstitial syndrome, it could lead to anterior pelvic tilt, increased lumbar lordosis, and abdominal distension. This state induces an excess load to the joints between the lumbar vertebrae and possibly leads to back pain (16).

Akuthota and Nadler (2004) showed in elderly women that the effect of the central stability training program is effective on their balance and postural stability (26); studies also showed that corrective exercises can help to stabilize posture and balance (6).

\section{CONCLUSION}

People with advanced MS have weakness in the abdominal muscles and loss of control of urine and stool. This weakness in the muscles can lead to anterior pelvic tilt, increased lumbar lordosis, 
and abdominal distension. The results of this study appear to be a good guide for designing and implementing motor and spots programs for people with MS. Focusing on balancing exercises that help improve their daily activities and exercise, is of outstanding points of the present study. More research is needed in the future to provide sports coaches with more practical information by intervening with a balance program and post-modification for MS patients.

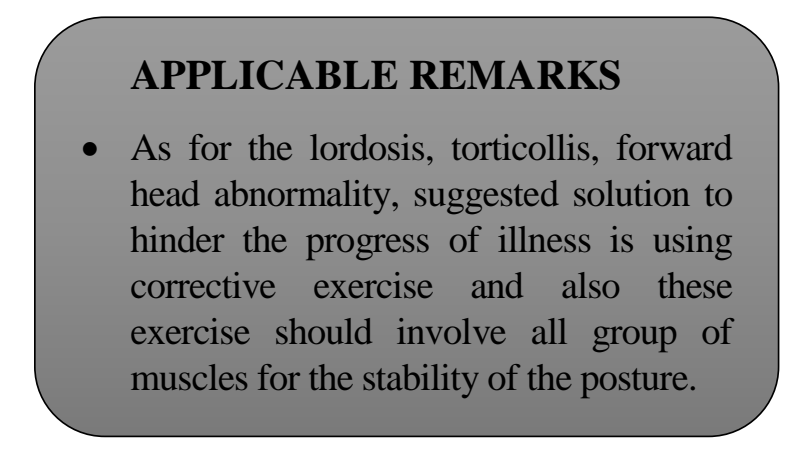

\section{REFERENCES}

1. Cabrera-Gómez JA, Kurtzke JF, González-Quevedo A, Lara-Rodriguez R. An epidemiological study of neuromyelitis optica in Cuba. Journal of neurology. 2009;256(1):35-44.

2. Donovan M, Sands J, Neighbors M, Marek J. Phipps medical-surgical nursing: Health and illness perspective. Philadelphia: Churchill Livingstone. 2003.

3. Clark M, Lucett S. NASM essentials of corrective exercise training: Lippincott Williams \& Wilkins; 2010.

4. Peterson-Kendall F, Kendall-McCreary E, Geise-Provance P, McIntyre-Rodgers M, Romani W. Muscles testing and function with posture and pain. Philadelphia: Lippincott Williams \& Wilkins; 2005.

5. Sluder JA, Newhouse P, Fain D. Pediatric and adolescent multiple sclerosis. Adolescent Medicine Clinics. 2002;13(3):461.

6. Ebrahimi HA, Sedighi B. Prevalence of multiple sclerosis and environmental factors in Kerman province, Iran. Neurology Asia. 2013;18(4):385-9.

7. Marrie RA. Environmental risk factors in multiple sclerosis aetiology. The Lancet Neurology. 2004;3(12):709-18.

8. Pazokian M, Shaban M, Zakerimoghdam M, Mehran A, Sanglaje B. The effect of stretching together aerobic exercises on fatigue level in multiple sclerosis patients refer to MS society of Iran those suffer from fatigue. Journal of Holistic Nursing And Midwifery. 2012;22(2):18-24.

9. Boes MK, Sosnoff JJ, Socie MJ, Sandroff BM, Pula JH, Motl RW. Postural control in multiple sclerosis: effects of disability status and dual task. Journal of the neurological sciences. 2012;315(1):44-8.

10. Guner S, Inanici F. Yoga therapy and ambulatory multiple sclerosis assessment of gait analysis parameters, fatigue and balance. Journal of bodywork and movement therapies. 2015;19(1):72-81.

11. de Vasconcelos GAR, Fernandes PRB, de Oliveira DA, Cabral ED, da Silva LVC. Avaliação postural da coluna vertebral em escolares surdos de 7-21 anos. Fisioterapia em Movimento. 2017;23(3).

12. Yahia A, Ghroubi S, Mhiri C, Elleuch M. Relationship between muscular strength, gait and postural parameters in multiple sclerosis. Annals of physical and rehabilitation medicine. 2011;54(3):144-55.

13. Van Emmerik R, Remelius J, Johnson M, Chung L, Kent-Braun J. Postural control in women with multiple sclerosis: effects of task, vision and symptomatic fatigue. Gait \& posture. 2010;32(4):608-14.

14. Sosnoff JJ, Shin S, Motl RW. Multiple sclerosis and postural control: the role of spasticity. Archives of physical medicine and rehabilitation. 2010;91(1):93-9.

15. Soyuer F, Mirza M, Erkorkmaz Ü. Balance performance in three forms of multiple sclerosis. Neurological research. 2006;28(5):555-62.

16. Horvat M, Ray C, Croce R, Blasch B. A comparison of isokinetic muscle strength and power in visually impaired and sighted individuals. Isokinetics and exercise science. 2004;12(3):179-83.

17. Gutierrez GM, Chow JW, Tillman MD, McCoy SC, Castellano V, White LJ. Resistance training improves gait kinematics in persons with multiple sclerosis. Archives of physical medicine and rehabilitation. 2005;86(9):18249.

18. Huisinga JM, Yentes JM, Filipi ML, Stergiou N. Postural control strategy during standing is altered in patients with multiple sclerosis. Neuroscience letters. 2012;524(2):124-8.

19. Mullen AE, Wilmarth MA, Lowe S. Cervical disk pathology in patients with multiple sclerosis: two case reports. Physical therapy. 2012;92(8):1055-64.

20. McLean SM, May S, Klaber-Moffett J, Sharp DM, Gardiner E. Risk factors for the onset of non-specific neck pain: a systematic review. Journal of Epidemiology \& Community Health. 2010;64(7):565-72.

21. Kasser SL, Jacobs JV. Understanding and treating balance impairment in multiple sclerosis. JCOM. 2014;21(9):419-32. 
22. Silva AG, Punt TD, Johnson MI. Reliability and validity of head posture assessment by observation and a fourcategory scale. Manual therapy. 2010;15(5):490-5.

23. Khalkhali M, Parnianpour M, Karimi H, Mobini B, Kazemnejhad A. The validity and reliability of measurement of thoracic kyphosis using flexible ruler in postural hyper-kyphotic patients. Journal of Biomechanics. 2006;39:S541.

24. Rajabi R, Samadi H. Laboratory guide corrective exersice. Tehran, Iran: Tehran University; 2009.

25. Schapiro RT. Managing symptoms of multiple sclerosis. Neurologic clinics. 2005;23(1):177-87.

26. Akuthota V, Nadler SF. Core strengthening1. Archives of physical medicine and rehabilitation. 2004;85:86-92. 